\title{
Heartbeat: the global burden of stroke due to untreated hypertension
}

Hypertension is a world-wide health burden that increases the risk of adverse cardiovascular outcomes with considerable geographical variation in awareness of the condition, appropriate treatment and blood pressure control. In this issue of Heart, O’Donnell and colleagues ${ }^{1}$ present the results of a standardised case-control study in 32 countries with over 13000 cases of acute first stroke matched to controls by age, sex and site. The association of hypertension with stroke was highest in countries with a lower gross national income and higher in younger versus older patients (figure 1). Compared with those with treated hypertension, untreated hypertension was associated with a higher OR for stroke incidence, younger age at first stroke, risk of intracerebral haemorrhage versus ischaemic stroke.

In an editorial, Sarfo ${ }^{2}$ summarises the concept of population attributable risk (PAR) of hypertension for stroke in high income countries compared with low- and middle-income countries : the PAR of aware and treated hypertension for stroke was $22.2 \%$ vs $17.3 \%$, aware but untreated was $4.8 \%$ vs $20.4 \%$ and unaware of hypertension was $5.6 \%$ vs 15.9\%'. Although PAR reflects the proportion of stoke attributable to hypertension, Sarfo points outs that complete elimination of hypertension is unlikely so the PAR probably overestimates the potential benefit of better treatment. Even so, he concludes: 'The time is rife for policymakers, providers and individuals to develop actionable policies and behavioural alterations in response to the reported associations between gaps in knowledge, awareness, and treatment of hypertension and stroke occurrence. The time for a concerted global effort to prevent the disability, dementia and deaths arising from stroke due to uncontrolled hypertension is now.'

Also in this issue, Ramlakhan and colleagues $^{3}$ present encouraging data on pregnancy outcomes in 202 women with

Division of Cardiology, University of Washington, Seattle, Washington, USA

Correspondence to Professor Catherine M Otto, Division of Cardiology, University of Washington, Seattle, WA 98195, USA; cmotto@uw.edu
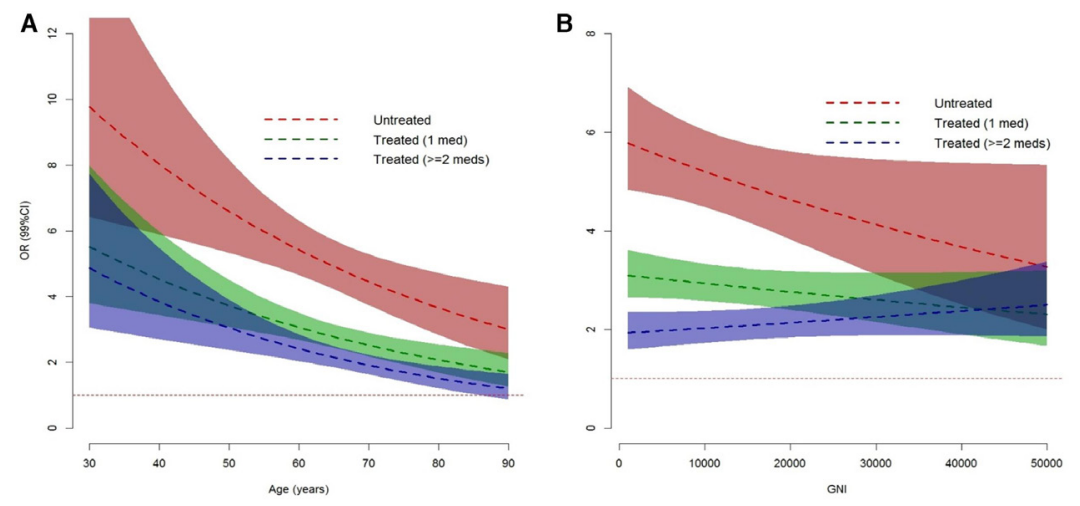

Figure 1 Figures report the association of hypertension with stroke by age (A) and GNI (B), demonstrating an increased slope in magnitude of association of hypertension with stroke by reducing age and reducing $\mathrm{GNI}$, which is modified by treatment status. Within increasing intensity of antihypertensive therapy, there is a diminution in slope of curve. While a gradient remains for risk of stroke by age among treatment groups, there is an inversion of gradient by GNI. These figures illustrate that increased uptake of antihypertensive therapy are expected to have greatest impact in younger populations and in lower-income regions. Multivariable model including age, smoking, waist-to-hip ratio , diabetes, physical activity, alternate healthy eating index, alcohol intake, psychosocial factors, apolipoproteins and cardiac risk factors. GNI, gross national income; PAR, population attributable risk. aortic coarctation (CoA). Although 9.6\% of these women had unrepaired aortic coarctation and $27.1 \%$ had pre-existing hypertension, there were no maternal deaths or aortic dissections. Only 4.3\% of women experienced a major adverse cardiovascular event, primarily heart failure. Premature birth occurred in 9.1\%

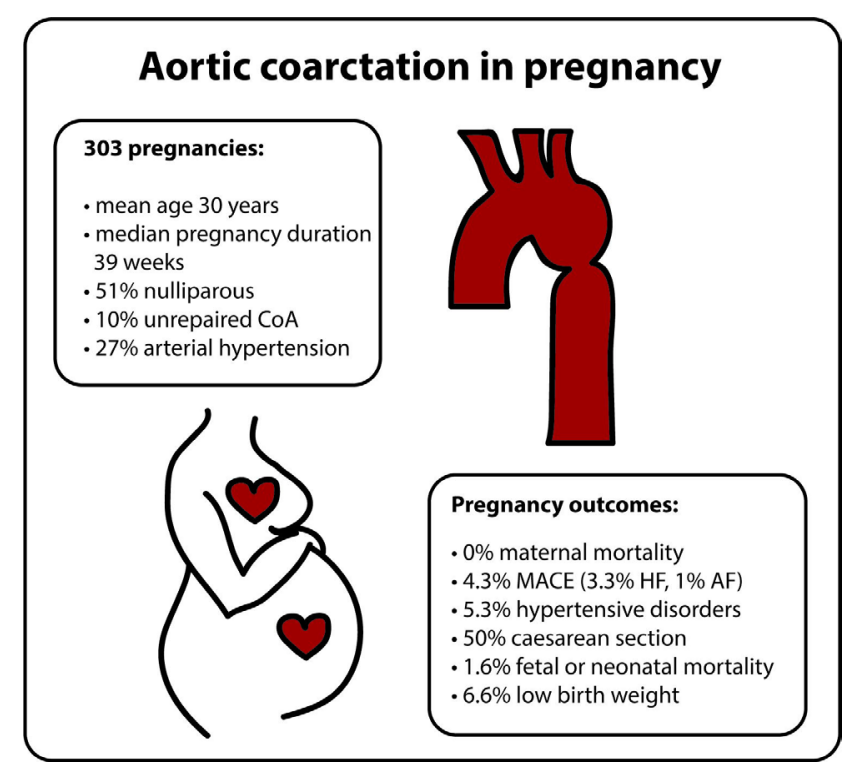

Figure 2 Summarising figure of pregnancy outcomes in women with aortic coarctation. AF, atrial fibrillation; CoA, aortic coarctation; HF, heart failure; MACE, major adverse cardiac event (defined as maternal cardiac death, $\mathrm{HF}, \mathrm{AF}$ or atrial flutter, ventricular tachyarrhythmia, endocarditis, thromboembolic events, aortic dissection and acute coronary syndrome). 
with four neonatal deaths, three of which were related to extreme premature birth (figure 2).

In the accompanying editorial, Cordina and $\mathrm{Li}^{4}$ review the pathophysiology of aortic coarctation and the potential risks associated with pregnancy including aortic dissection, hypertension, diastolic heart failure, intracranial haemorrhage and aortic valve dysfunction, as well as obstetrical complications. They conclude: '(in accordance with current guidelines) that a woman with coarctation but no major comorbidities and an aorta with minimal obstruction and diameter $<40 \mathrm{~mm}$, good functional class and left ventricular ejection $>40 \%$ is at low risk for maternofetal complications but these new data are not sufficient for us to let our guard down in women with high risk features.'

A review article in this issue provides recommendations on the role of echocardiography in screening and evaluation of athletes for prevention of sudden cardiac death (SCD) (figure 3). Niederseer and colleagues ${ }^{5}$ propose that 'first echocardiography is performed during adolescence to rule out structural heart conditions associated with SCD that cannot be detected by ECG, especially mitral valve prolapse, coronary artery anomalies, bicuspid aortic valve and dilatation of the aorta. A second echocardiography could be performed from the age of 30-35 years, when athletes age and become master athletes, to especially evaluate pathological cardiac remodelling to exercise (eg, atrial and/ or right ventricular dilation), late onset cardiomyopathies and wall motion abnormalities due to myocarditis or coronary artery disease.'

A counterpoint is provided by Dineen and Prutkin ${ }^{6}$ who argue that: 'the echocardiogram has a significant role in the secondary evaluation of abnormal history, physical and ECG findings but we do not think it should be used widely for initial screening. We agree that it can pick up structural heart conditions that will need long-term follow-up. However, the purpose of athletic screening should be to pick up life-threatening conditions which could lead to SCD when triggered by exercise or else we would recommend screening all adolescents and adults. Until we have more concrete evidence, we believe the screening echocardiogram does not appear to improve SCD risk stratification in athletes more than the current standard of practice.'

Another provocative review article by Grayburn and colleagues ${ }^{7}$ provides perspective on the optimal definition for severity of secondary mitral regurgitation

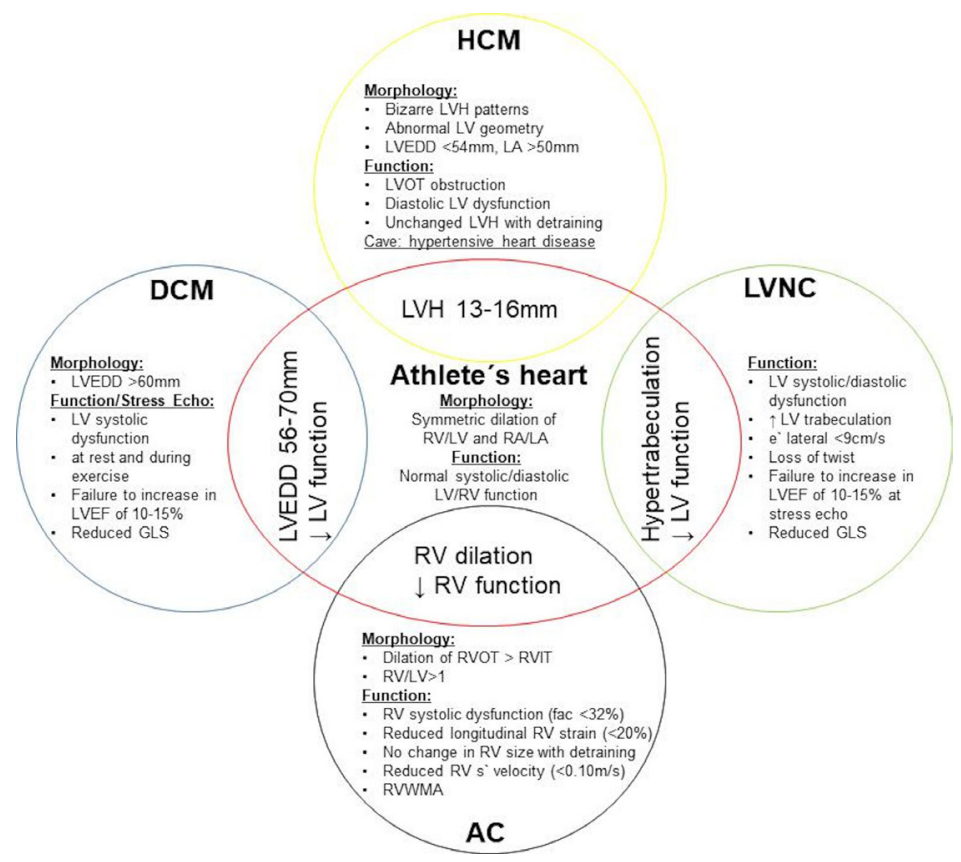

Figure 3 Differentiating athlete's heart from HCM, DCM, AC and LVNC. AC, arrhythmogenic cardiomyopathy; DCM, dilated cardiomyopathy; GLS, global longitudinal strain; HCM, hypertrophic cardiomyopathy; LA, left atrium; LV, left ventricle; LVEDD, left ventricular end-diastolic diameter; LVEF, left ventricular ejection fraction; LVH, left ventricular hypertrophy; LVNC, left ventricular non-compaction; LVOT, left ventricular outflow tract; RA, right atrium; RV, right ventricle; RVIT, right ventricular inflow tract; RVOT, right ventricular outflow tract; RVWMA, right ventricular wall motion abnormalities.

(SMR). The ratio of effective regurgitant orifice area (EROA) to left ventricular (LV) end-diastolic volume (EDV) can be useful and may explain the difference outcomes in clinical trials of mitral transcatheter edge-to-edge repair (TEER) but the EROA/ LVEDV ratio still fails to consider other

important factors (figure 4). They recommend: 'the key to patient selection is forced titration of neurohormonal antagonists to the target doses that have been proven in clinical trials (along with cardiac resynchronisation when appropriate). Patients who continue to have symptomatic severe

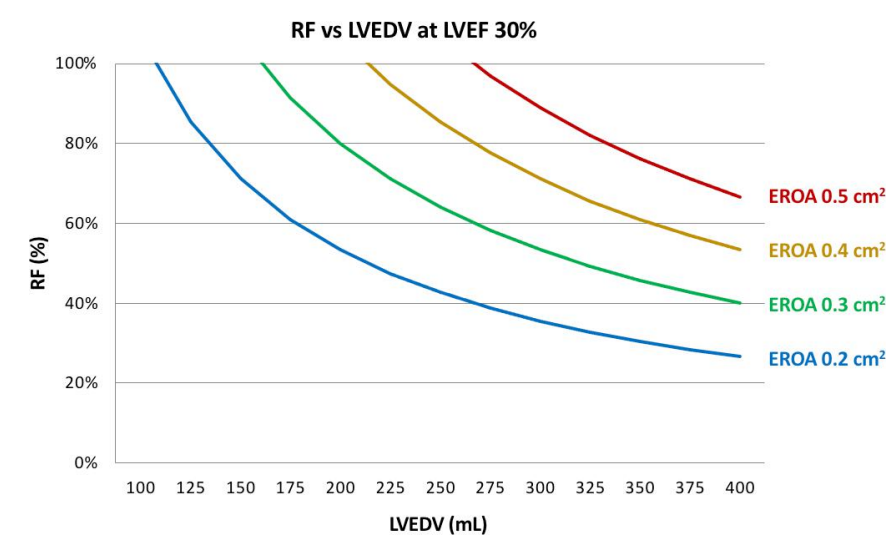

Figure 4 Plot showing the relationship of RF ( $x$-axis) versus LVEDV (y-axis) for different values of EROA and an LVEF (30\%). At this low LVEF, RF would be 100\% (physiologically impossible) at LVEDV of approximately $275 \mathrm{~mL}$ at a true (mean over systole per the Gorlin hydraulic orifice area) EROA $0.5 \mathrm{~cm}^{2}, 220 \mathrm{~mL}$ at an EROA $0.4 \mathrm{~cm}^{2}, 160 \mathrm{~mL}$ at an EROA $0.3 \mathrm{~cm}^{2}$ and $115 \mathrm{~mL}$ at an EROA $0.2 \mathrm{~cm}^{2}$. Peak EROA values reported by single frame echocardiographic techniques are often physiologically impossible. It is important to recognise the difference between peak EROA and true EROA. Regurgitant volume obtained by multiplying peak EROA by the velocity-time integral of MR will often result in physiologically impossible values. EROA, effective regurgitant orifice area; LVEDV, left ventricular end-diastolic volume; LVEF, left ventricular ejection fraction; RF, regurgitant fraction. 

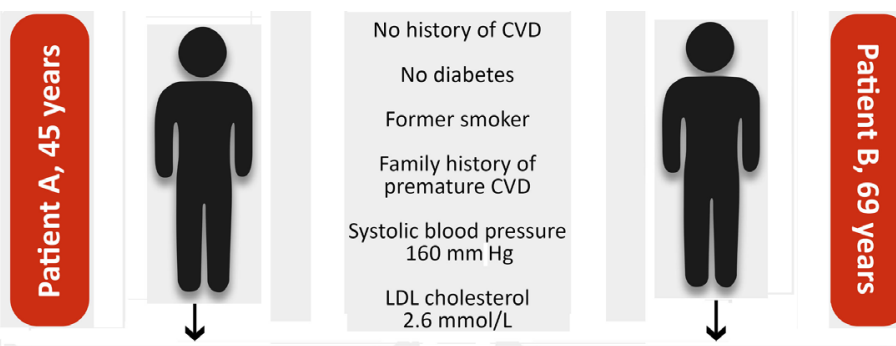

Absolute 10-year risk $1 \%$

\section{CVD-free life expectancy}

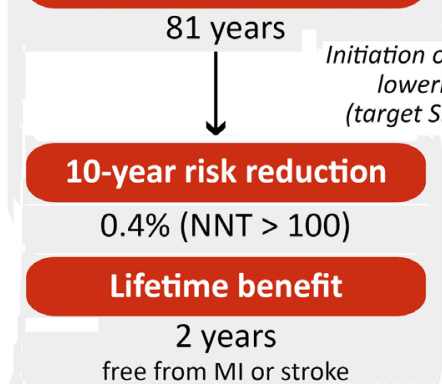

Absolute 10-year risk

$9 \%$

CVD-free life expectancy

85 years of blood pressure
ving therapy

$140 \mathrm{~mm} \mathrm{Hg}$ )

\begin{tabular}{|c|}
\hline 10-year risk reduction \\
\hline $3.9 \%$ (NNT 26) \\
\hline Lifetime benefit \\
1 year \\
free from Ml or stroke
\end{tabular}

Figure 5 Patient example: 10-year risk and treatment effects compared with the CVD-free life expectancy and lifetime benefit for a younger versus an older individual. Ten-year predictions were estimated using the Systemic Coronary Risk Estimation model risk model; lifetime predictions using the LIFEtime-perspective CardioVascular Disease model. CVD, cardiovascular disease. LDL, low density lipoprotein; NNT, number needed to treat: MI, myocardial infarction; SBP, systolic blood pressure

SMR after doing so should be considered for TEER.' This recommendation is in accord with the recently published 2020 American College of Cardiology/American Heart Association Guidelines for the Management of Valvular Heart disease. ${ }^{8}$

In contrast, Kamoen and colleagues ${ }^{9}$ argue that the concept of proportionality between SMR severity and LV enddiastolic volumes remains hypothetical and requires validation in clinical trials. In addition, both articles emphasise the technical challenges and measurement variability which affect these echocardiographic parameters. Perhaps we need to consider alternative, possibly more accurate and robust, measures of SMR severity. ${ }^{10}$
The Education in Heart article in this issue $^{11}$ reviews several cardiovascular risk prediction tools and provides guidance on which score is best suited to each patient (figure 5). Examples are provided for the effects of risk reduction therapy for individuals with different 10 -year risk scores, showing that absolute risk scores need to be interpreted in terms of lifetime benefit.

Funding The authors have not declared a specific grant for this research from any funding agency in the public, commercial or not-for-profit sectors.

Competing interests None declared.

Patient and public involvement Patients and/ or the public were not involved in the design, or conduct, or reporting, or dissemination plans of this research.

Patient consent for publication Not required.
Provenance and peer review Commissioned; internally peer reviewed.

(C) Author(s) (or their employer(s)) 2021. No commercial re-use. See rights and permissions. Published by BMJ.

\section{(A) Check for updates}

To cite Otto CM. Heart 2021;107:259-261.

Heart 2021;107:259-261.

doi:10.1136/heartjnl-2021-318954

ORCID iD

Catherine M Otto http://orcid.org/0000-0002-05279392

\section{REFERENCES}

1 O' Donnell M, Hankey GJ, Rangarajan S. Variations in knowledge, awareness and treatment of hypertension and stroke risk by country income level. Heart 2021;107:282-9.

2 Sarfo FS. Associations between hypertension knowledge, awareness, and treatment and stroke occurrence across the globe: time to act on what we know. Heart 2021;107:264-5.

3 Ramlakhan KP, Tobler D, Greutmann M. Pregnancy outcomes in women with aortic coarctation. Heart 2021;107:290-8.

4 Cordina R, Li W. Pregnancy with coarctation appears low risk overall but individual cardiovascular evaluation remains essential. Heart 2021;107:266-7.

5 Niederseer D, Rossi VA, Kissel C. Role of echocardiography in screening and evaluation of athletes. Heart 2021;107:270-6.

6 Dineen E, Prutkin J. Counterpoint: lack of evidence for echocardiography screening in athletes. Heart 2021;107:262-3.

7 Grayburn PA, Packer M, Sannino A, et al. Disproportionate secondary mitral regurgitation: myths, misconceptions and clinical implications. Heart 2020. doi:10.1136/heartjnl-2020-316992. [Epub ahead of print: 24 Nov 2020].

8 Otto CM, Nishimura RA, Bonow RO, et al. 2020 ACC/AHA guideline for the management of patients with valvular heart disease: a report of the American College of Cardiology/American heart association joint Committee on clinical practice guidelines. Circulation 2020:CIR0000000000000923.

9 Kamoen V, Calle S, De Buyzere M, et al. Proportionate or disproportionate secondary mitral regurgitation: how to untangle the Gordian knot? Heart 2020;106:1719-25.

10 Kusunose $\mathrm{K}$. What is a better measure of regurgitant severity in secondary mitral regurgitation by echocardiography? Heart 2020;106:874-5.

11 de Vries TI, Visseren FLJ. Cardiovascular risk prediction tools made relevant for GPs and patients. Heart 2021;107:332-40. 